\title{
Recent Results using the DANCE Detector at Los Alamos
}

\author{
J.L. Ullmann ${ }^{1}$, R. Reifarth ${ }^{2 *}$, A.J. Couture ${ }^{1}$, R.C. Haight ${ }^{1}$, J.M. O'Donnell ${ }^{1}$, T.A. \\ Bredeweg $^{1}$, E.M. Bond ${ }^{1}$, E.P. Chamberlin ${ }^{1}$, M.M. Fowler ${ }^{1,3}$, M. Jandel ${ }^{1}$, A. \\ Kronenberg $^{1}$, R.S. Rundberg ${ }^{1}$, D.J. Vieira ${ }^{1}$, J.B. Wilhelmy ${ }^{1}$, J.M. Wouters ${ }^{1}$, E.-I. \\ Esch $^{1}$, D.D. Strottman ${ }^{1}$, U. Agvaanluvsan ${ }^{3}$, J.A. Becker ${ }^{3}$, R. Macri ${ }^{3}$, W. Parker ${ }^{3}$, P. \\ Wilk $^{3}$, C.Y. Wu ${ }^{3}$, U. Greife ${ }^{4}$, R. Hatarik ${ }^{4}$, A. Alpizar ${ }^{4}$, P.E. Koehler ${ }^{5}$, G.E. Mitchell ${ }^{6}$, \\ S.A. Sheets ${ }^{6}$, F. Kaeppeler ${ }^{7}$, M. Heil ${ }^{7}$ \\ ${ }^{1}$ Los Alamos National Laboratory, Los Alamos NM 87545, USA. \\ ${ }^{2}$ Gesellschaft fuer Schwerionenforschung, 64291 Darmstadt, Germany. \\ ${ }^{3}$ Lawrence Livermore National Laboratory, Livermore CA 94551, USA. \\ ${ }^{4}$ Colorado School of Mines, Golden, CO 80401, USA. \\ ${ }^{5}$ Oak Ridge National Laboratory, Oak Ridge TN 37830, USA. \\ ${ }^{6}$ North Carolina State Univerisity, Raleigh, NC 27695, USA \\ ${ }^{7}$ Forschungszentrum Karlsruhe, 76133 Karlsruhe, Germany. \\ E-mail: ullmann@lanl.gov \\ LA-UR-07-6004
}

The Detector for Advanced Neutron Capture Experiments (DANCE) is a 160 -element, $4 \pi \mathrm{BaF}_{2}$ array designed to make neutron-capture measurements on small quantities of rare or radioactive nuclides. The high segmentation also allows measurements of the gamma-ray multiplicity. This paper describes some preliminary results from capture cross section, gamma-ray multiplicity, and capture-to-fission measurements.

Workshop on Photon Strength Functions and Related Topics

Prague, Czech Republic

17-20 June 2007

* $\quad$ Speaker 


\section{Introduction}

Accurate neutron capture cross sections from thermal to hundreds of kilovolts are needed for many current applications, as well as for a basic understanding of neutron capture. Capture cross sections are difficult to calculate accurately a priori, and therefore must be measured. Many precise measurements of capture for stable nuclides have been made, but there are very few measurements on radioactive targets. For these nuclides, $1 \mathrm{mg}$ may equal 1 Curie or more of activity, introducing problems in sample preparation and handling. DANCE, the Detector for Advanced Neutron Capture Experiments, was designed to combine a high-efficiency detector with a high neutron flux to make precise measurements of neutron capture on samples of $1 \mathrm{mg}$, or less, of material.

\section{Detector and Flight Path}

DANCE was designed to be a calorimetric detector in which the total energy of the gamma-ray cascade following capture is measured. The total energy is equal to the $\mathrm{Q}$ value of the reaction plus the center-of-mass neutron energy, and can be used to help identify the capturing nucleus. The detector is a sphere of 162 equal-area segments of four different shapes. Two of the segments are empty to allow the beam to enter and exit. The detector segments are $15 \mathrm{~cm}$ long $\mathrm{BaF}_{2}$ crystals coupled to Electron Tubes model 9921 quartz-window phototubes. Barium fluoride was chosen to minimize events due to capture of scattered neutrons in the detector. A $6 \mathrm{~cm}$ thick ${ }^{6} \mathrm{LiH}$ sphere with inner radius of $10.5 \mathrm{~cm}$ surrounds the sample location to further reduce the scattered neutron background. The crystal faces are $18 \mathrm{~cm}$ from the center of the sphere, for a total solid angle of $3.52 \pi$. Barium fluoride has internal radioactivity of about $0.2 \mathrm{~Bq} / \mathrm{cm}^{3}$ from the decay chain of ${ }^{226} \mathrm{Ra}$, a chemical homologue of $\mathrm{Ba}$. This produces an alpha background of about 150 to 200 counts/sec in each crystal. Extensive Monte Carlo simulations were made to understand the design parameters of the detector ${ }^{1}$ and the various experimental backgrounds ${ }^{2}$.

DANCE was constructed on Flight Path 14 at the Manuel J. Lujan Jr. Neutron Scattering Center at the Los Alamos Neutron Science Center. The flight path views the partially-coupled second-tier "backscatter" water moderator. The sample location is at $20.33 \mathrm{~m}$ and the beam stop at $30 \mathrm{~m}$ from the moderator. Outside of the bulk shield surrounding the neutron production target, the beam pipes are made of aluminum and all collimation is copper in order to eliminate backgrounds from neutron capture on iron. The collimation was designed to provide a small ( $1 \mathrm{~cm}$ diameter) beam spot with minimal penumbra at the sample location.

The neutron flux is measured by three neutron monitors located $2 \mathrm{~m}$ downstream of the sample location. The monitors are a $\mathrm{BF}_{3}$ proportional counter, ${ }^{235} \mathrm{U}$ fission chamber, and $\mathrm{Si}$ surface-barrier detector viewing a ${ }^{6} \mathrm{LiF}$ deposit on a mylar film. The absolute integral value of the neutron flux was measured at the sample location by Au activation using a $2 \mathrm{mg} / \mathrm{cm}^{2} \mathrm{Au}$ foil with and without $\mathrm{Cd}$ according to the Westcotte convention. Figure 1 shows the neutron 
spectrum measured with the ${ }^{6} \mathrm{Li}(\mathrm{n}, \mathrm{t})$ reaction and normalized to the Au activation. Also shown is the flux calculated by Muhrer ${ }^{3}$ for the "as-built" configuration of the flight path.

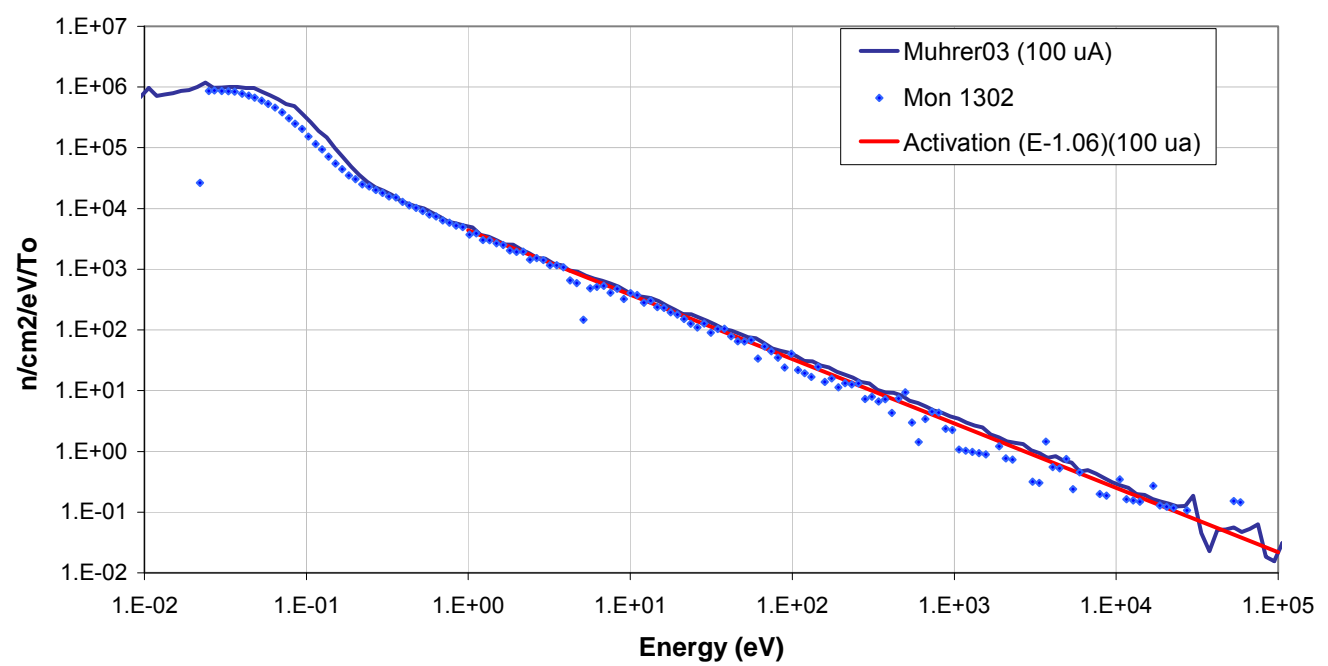

Figure 1. Neutron flux measured at the sample location in the DANCE detector, normalized to thin Au activation. The "activation" curve is a power-law $\left(E^{-1.06}\right)$ normalized to the activation measurement.

A typical event rate for a $4 \mathrm{mg} / \mathrm{cm}^{2} \mathrm{Au}$ target is 400 events in a $2 \mathrm{~ms}$ gate. The data acquisition system must handle multiple events per beam burst, fast digitization of the pulse heights, time measurements out to $14 \mathrm{~ms}$ with $5 \mathrm{~ns}$ or better time resolution, and rapid data transfer times. Fast waveform digitizers were chosen to meet these requirements. DANCE uses Acqiris DC-265 digitizers ${ }^{4}$, which are 8-bit, 500 megasample/sec, $150 \mathrm{MHz}$ bandwidth digitizers with 128 thousand memory points per channel. The phototube anode signal is read directly by the digitizers, and two digitizers per crystal are used. The digitizers are configured with 12 detectors (24 digitizers) in a compact PCI crate. A $1.8 \mathrm{GHz}$ single-board computer is mounted in each crate to control the digitizers and perform preliminary analysis of the pulse shape. The data acquisition system is described more fully in Ref 5 .

Understanding and controlling instrumental backgrounds is important for neutron capture measurements. There are several sources of background in DANCE, both beam related and beam independent. Many of these can be measured by making blank-target runs, and backgrounds from alpha decays in the $\mathrm{BaF}_{2}$ detectors can be eliminated by pulse-shape discrimination. However, at neutron energies above about $1 \mathrm{keV}$, events from neutrons scattering from the sample and subsequently capturing on Ba isotopes in the detector become an important source of background, even with the ${ }^{6} \mathrm{LiH}$ absorber. The scattering contribution can be estimated using a $\mathrm{C}$ or ${ }^{208} \mathrm{~Pb}$ target, for which the scattering cross section is much greater than the capture cross section. An additional externally-produced neutron background results 
from mistuning of the primary proton beam. This background is monitored by a ${ }^{6} \mathrm{Li}$-glass scintillator located on the flight-path shielding away from the neutron beam.

Another source of background is gamma rays produced in the neutron-production target and moderator region, and by neutrons capturing along the flight path ${ }^{2}$. These gammas interact by Compton scattering or pair production in the sample, and are then detected with high efficiency in DANCE. Since the interaction probability goes like $\mathrm{Z}$ for Compton scattering, and $\mathrm{Z}^{2}$ for pair production, this background is more important for heavy elments. It has been estimated by measuring gammas scattered from a $\mathrm{Pb}$ target with most of the neutrons stopped by a $\mathrm{CH}_{2}$ neutron absorber in the beam.

\section{Preliminary Results}

The experimental program at DANCE has three main components: Defense and Nonproliferation, Nuclear Power and Advanced Reactors, and Basic Science and Nuclear Astrophysics. Many measurements have been performed and data are nearing publication, but presented here will be some preliminary results from the different areas.

\subsection{Neutron capture on ${ }^{234} \mathrm{U}$}

Neutron capture on the actinides is of interest to both defense and non-proliferation programs and to nuclear power. There have been very few measurements on ${ }^{234} \mathrm{U}$, and this represents the first high-resolution measurement. The target consisted of $1.08 \mathrm{mg}$ of ${ }^{234} \mathrm{U}$ deposited on two $2.5 \mu \mathrm{m}$ Ti foils. The data were analyzed for multiplicites $\geq 3$ and summed gamma energies between 4.0 and $5.5 \mathrm{MeV}$. (The $\mathrm{Q}$ value for capture is $5.30 \mathrm{MeV}$.) The results are shown in Figure 2.

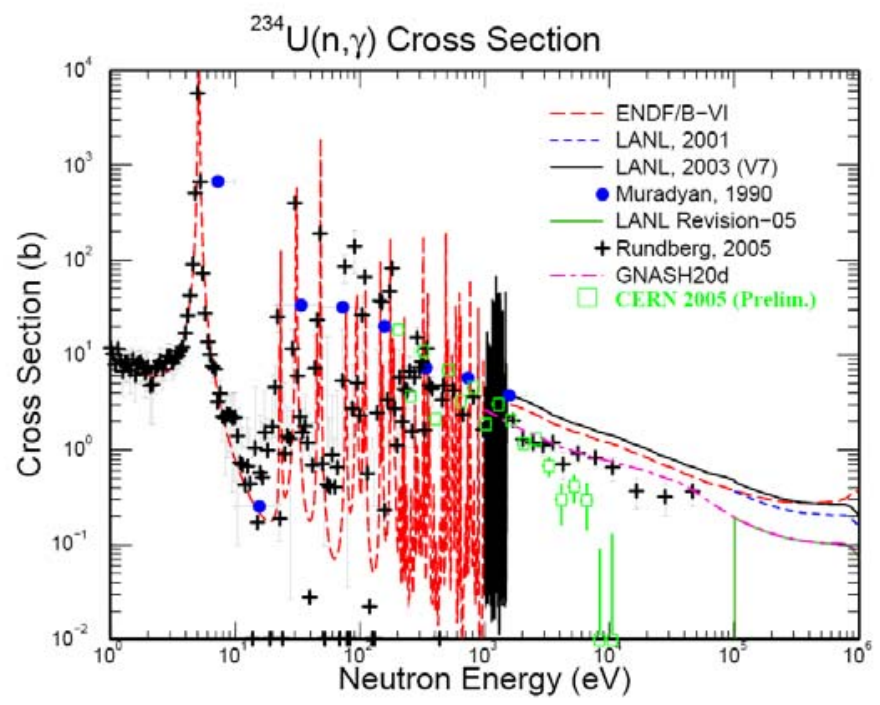

Figure 2. ${ }^{234} U(n, Y)$ measured at DANCE (labelled as Rundberg 2005) compared to other data and evaluations. 
Background subtractions corresponding to blank target (Ti foils only), ${ }^{234} \mathrm{U}$ fission, and scattering of beam gammas (about a 20\% effect) were made. The cross sections were normalized to the thermal value of $100 \mathrm{~b}$ and absolutely using the Barr method for determining efficiency. The integral of the $5.16 \mathrm{eV}$ resonance using the thermal normalization is $1770 \mathrm{~b}-\mathrm{eV}$, and using the Barr normalization is $1740 \mathrm{~b}-\mathrm{eV}$. This compares to a value of $2830 \mathrm{~b}-\mathrm{eV}$ from the ENDF/B-VI evaluation. The data in the continuum region are also below the previous evaluation. The END/B-VII evaluation ${ }^{6}$ adopts this lower cross section.

\subsection{Capture to fission ratios on fissile nuclides}

The capture-to-fission ratio on nuclides such as ${ }^{235} \mathrm{U}$ and ${ }^{239} \mathrm{Pu}$ is also of interest to both defense and reactor programs. A fission-tagging detector is needed to separate gammas following fission from those due to capture. For DANCE, we have developed a $5 \mathrm{~cm}$ diameter parallel-plate avalanche counter ${ }^{7}$ to fit inside the DANCE beam pipe. Low-energy resonance data on ${ }^{235} \mathrm{U}$ from a test run are shown in Figure 3. The sample consisted of $460 \mu \mathrm{g}$ of $99.89 \%$ pure ${ }^{235} \mathrm{U}$ electrodeposited on a metalized mylar foil in a $0.7 \mathrm{~cm}$ diameter deposit. The capture data were analyzed for multiplicity $\geq 4$ and summed gamma energy from 5.5 to $7.5 \mathrm{MeV}$. The fission tagging was $78 \%$ efficient, and the capture spectrum was corrected for fission contamination. A production run has been completed and is under analysis. An extensive program on capture/fission measurements is planned. For more information, see Ref. 7.

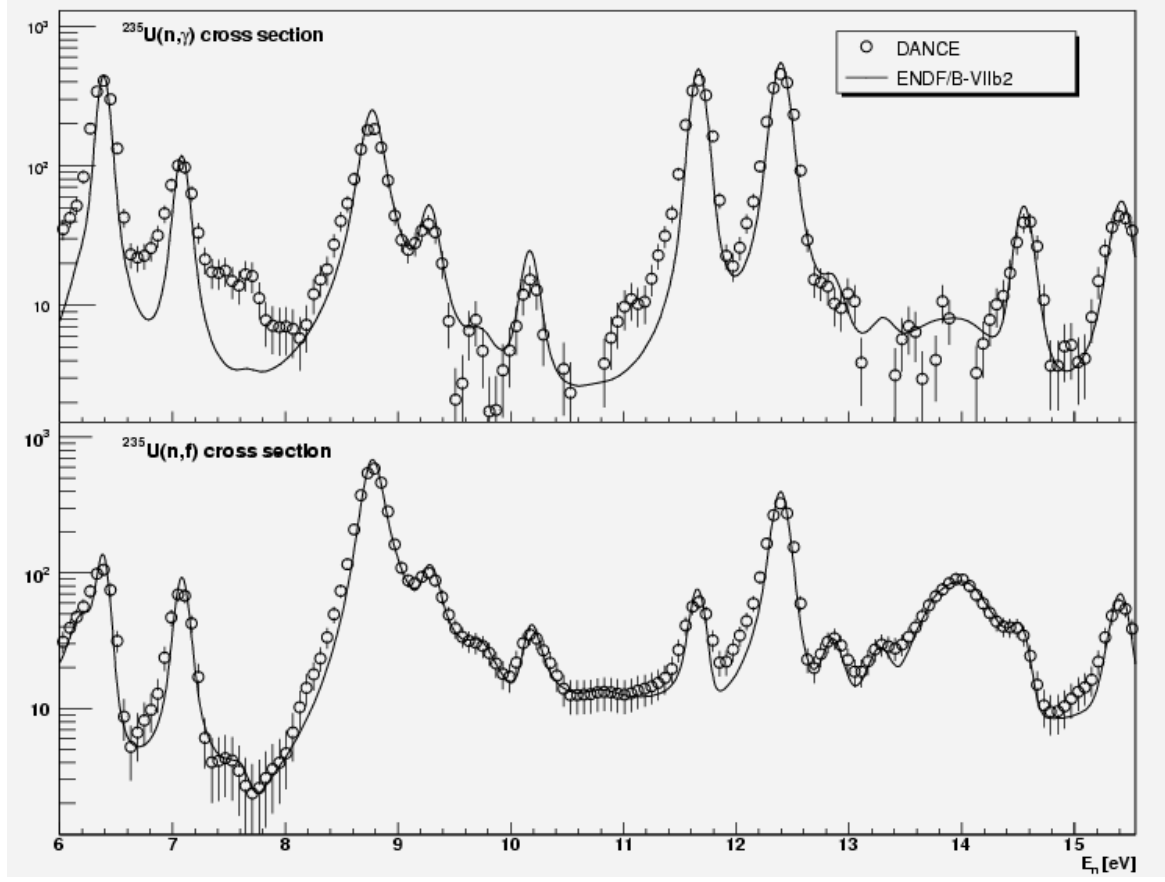

Figure 3. Preliminary ${ }^{235} U$ capture and fission data using the fission-tagging PPAC. 


\section{$3.3 \quad{ }^{237} \mathrm{~Np}$ Capture}

Measurements of capture on ${ }^{237} \mathrm{~Np}$ were made to resolve discrepencies in previous measurements. The sample consisted of $0.44 \mathrm{mg}$ of ${ }^{237} \mathrm{~Np}$ (freshly separated to remove ${ }^{233} \mathrm{~Pa}$ ) electroplated in a $6.4 \mathrm{~mm}$ diameter deposit on two $2 \mu \mathrm{m}$ thick Ti foils. The foils were glued together with the deposits facing each other, and mounted in a radioactive target holder with 50 $\mu \mathrm{m}$ kapton windows. The data set was normalized to the $0.5 \mathrm{eV}$ resonance ${ }^{8}$. The data in the $\mathrm{keV}$ region are shown in figure 4 and compared to other recent measurements. Also shown are calculations of the cross section made by Kawano using the code $\mathrm{CoH}$. The data are represented best by calculations using $\left\langle\Gamma_{\gamma}>=0.0312 \mathrm{eV}\right.$. For more details, see Ref. 8 .

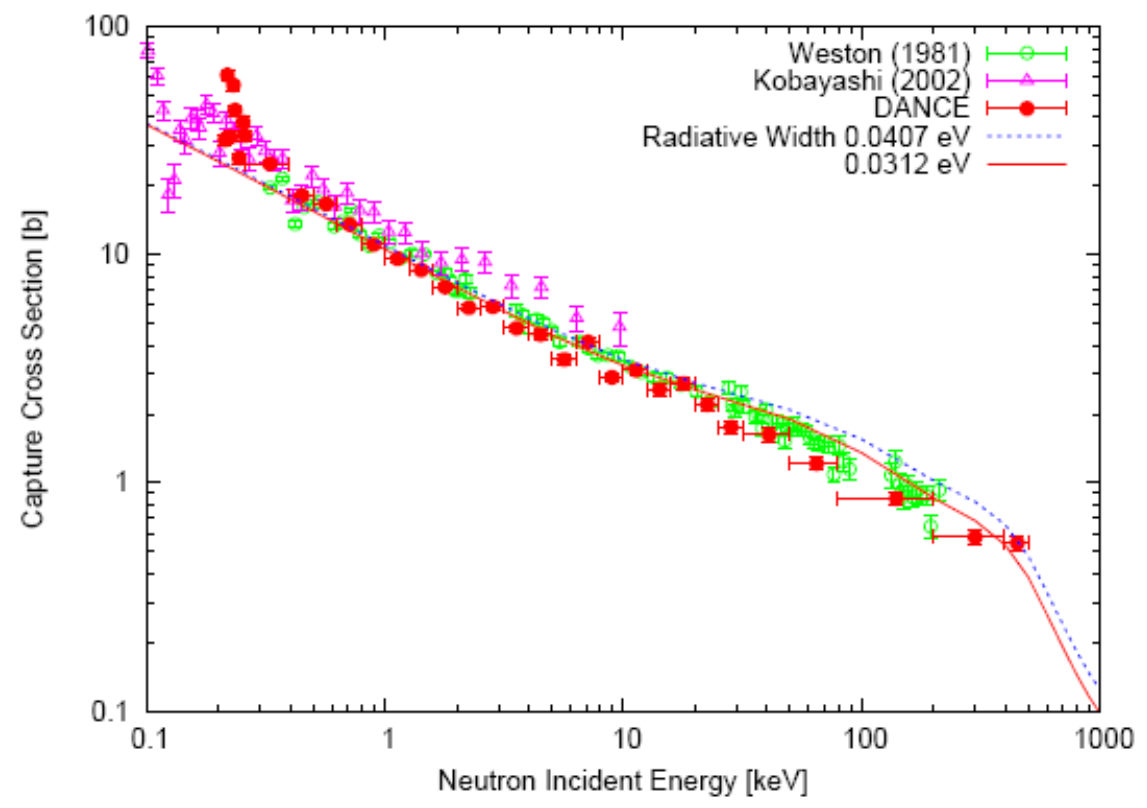

Figure 4. The ${ }^{237} N p$ data from DANCE compared to other recent measurements. Also shown are calculations made by Kawano using the code CoH. (From Ref. 8.)

\subsection{Gamma-ray multiplicity of resonances and spin}

As an example of basic-physics research, the gamma-ray multiplicity from capture in resonances can be used to determine the spin $\mathrm{J}$ of the resonance in certain nuclei ${ }^{9}$. An experiment on ${ }^{147} \mathrm{Sm}(\mathrm{n}, \gamma)$ was done by Koehler ${ }^{11}$ to determine resonance spins to resolve discrepencies observed in a previous ${ }^{147} \mathrm{Sm}(\mathrm{n}, \alpha)$ experiment ${ }^{10}$. Metallic targets of $97.93 \%$ pure metallic ${ }^{147} \mathrm{Sm}$ up to $10 \mathrm{mg} / \mathrm{cm}^{2}$ thick were used. An example of the data in the region 50 to 150 $\mathrm{eV}$ is shown in figure 5. Figure 6 shows the "cluster multiplicity" distribution for two resonances with firm $\mathrm{J}^{\pi}$ assignments. The $\mathrm{J}=4$ resonance at $39.7 \mathrm{eV}$ has an average multiplicity $<\mathrm{M}>=4.45$, and the $\mathrm{J}=3$ resonance at $40.7 \mathrm{eV}$ has $<\mathrm{M}>=4.11$. 


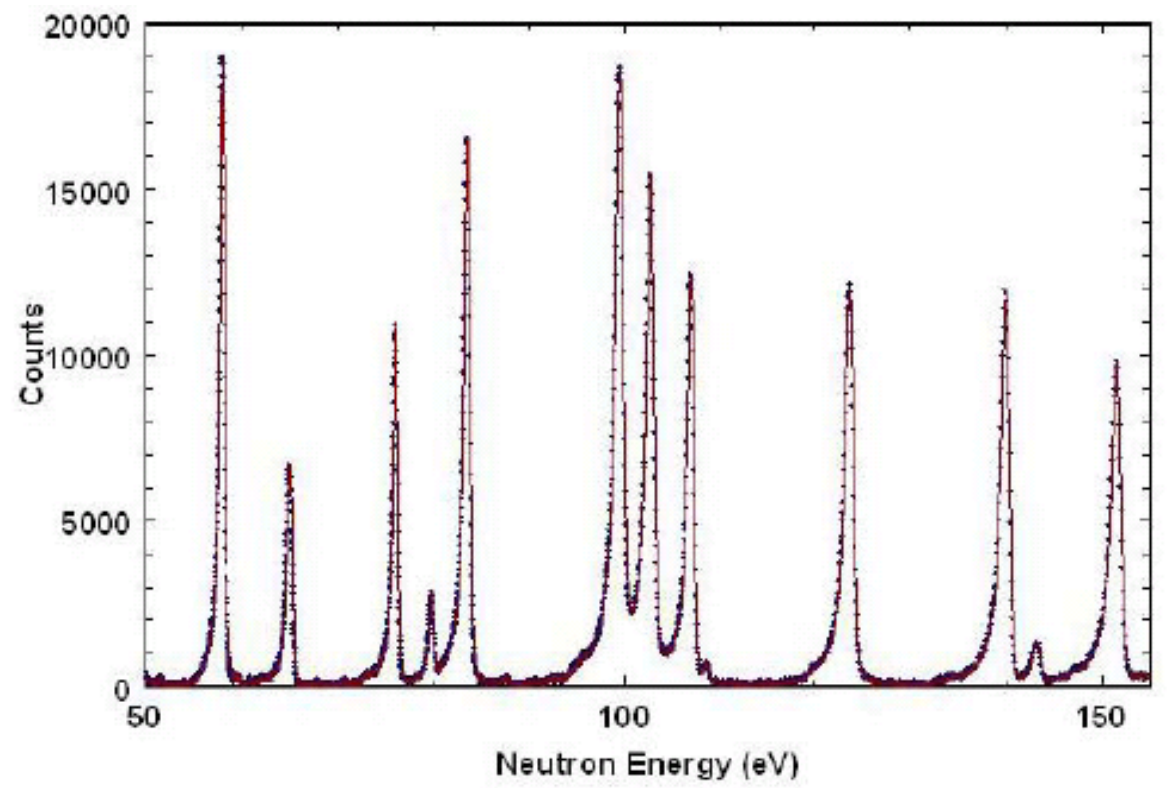

Figure 5. Neutron capture data for ${ }^{147} \mathrm{Sm}(n, \gamma)$ for neutron energies from 50 to $150 \mathrm{eV}$.

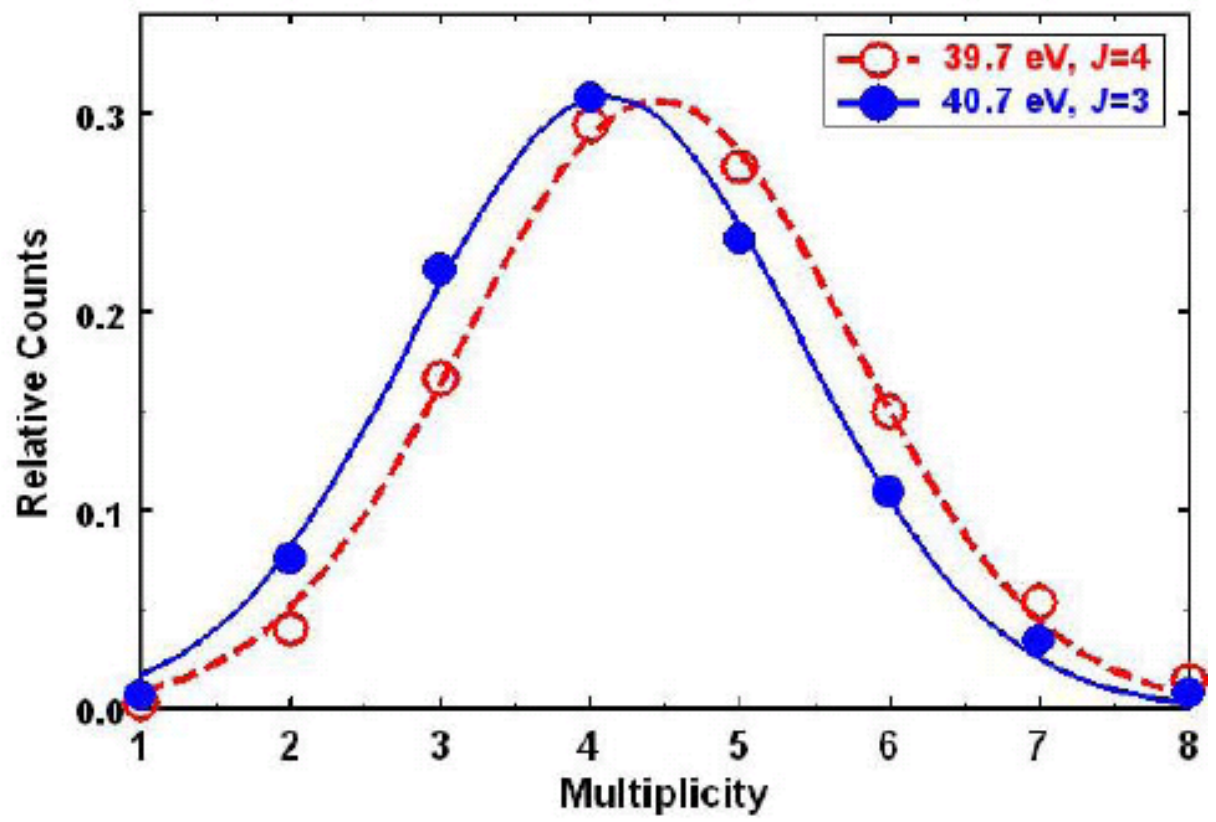

Figure 6. Cluster multiplicity distribution for two resonances with known spin 
The actual spin assignments were made using a polynomial technique. Spin assignments were made for 140 resonances below $1 \mathrm{keV}$. There were 34 new firm $\mathrm{J}$ assignments, an additional 8 firm assignments where only tentative values were known, 6 firm assignments that disagreed with compilations, 6 previously firm assignmens were shown in fact to be doublets, and 14 resonances were left without firm assignments. The result of the analysis was an indication of possible non-statistical (non-Porter-Thomas) distributions for the reduced neutron widths of the resonances. For more information, see ref. 11.

\subsection{Monte Carlo simulations}

A detailed understanding of the response of the DANCE ball is needed for absolute crosssection measurements as well for studies of photon strength functions. Monte Carlo studies ${ }^{12,13}$ have shown that the cluster multiplicity measured in DANCE is a good approximation to the true gamma-ray multiplicity. Recent studies have used $\mathrm{Y}$-ray cascades estimated using the program DICEBOX ${ }^{14}$ as input to a model of the DANCE array in GEANT- $4^{15}$ to study the response of the ball to the gamma-ray spectrum from different multipolarity resonances ${ }^{12}$. An example of the results of these studies is shown in Figure 7, which shows the efficiency as a function of cluster multiplicity for the $0.3 \mathrm{eV} 2$ - and $1.27 \mathrm{eV} 3$ - states observed in ${ }^{241} \mathrm{Am}(\mathrm{n}, \mathrm{Y})$. The Monte Carlo calculations are in excellent agreement with the measured distributions. For more details, see Ref. 12 .
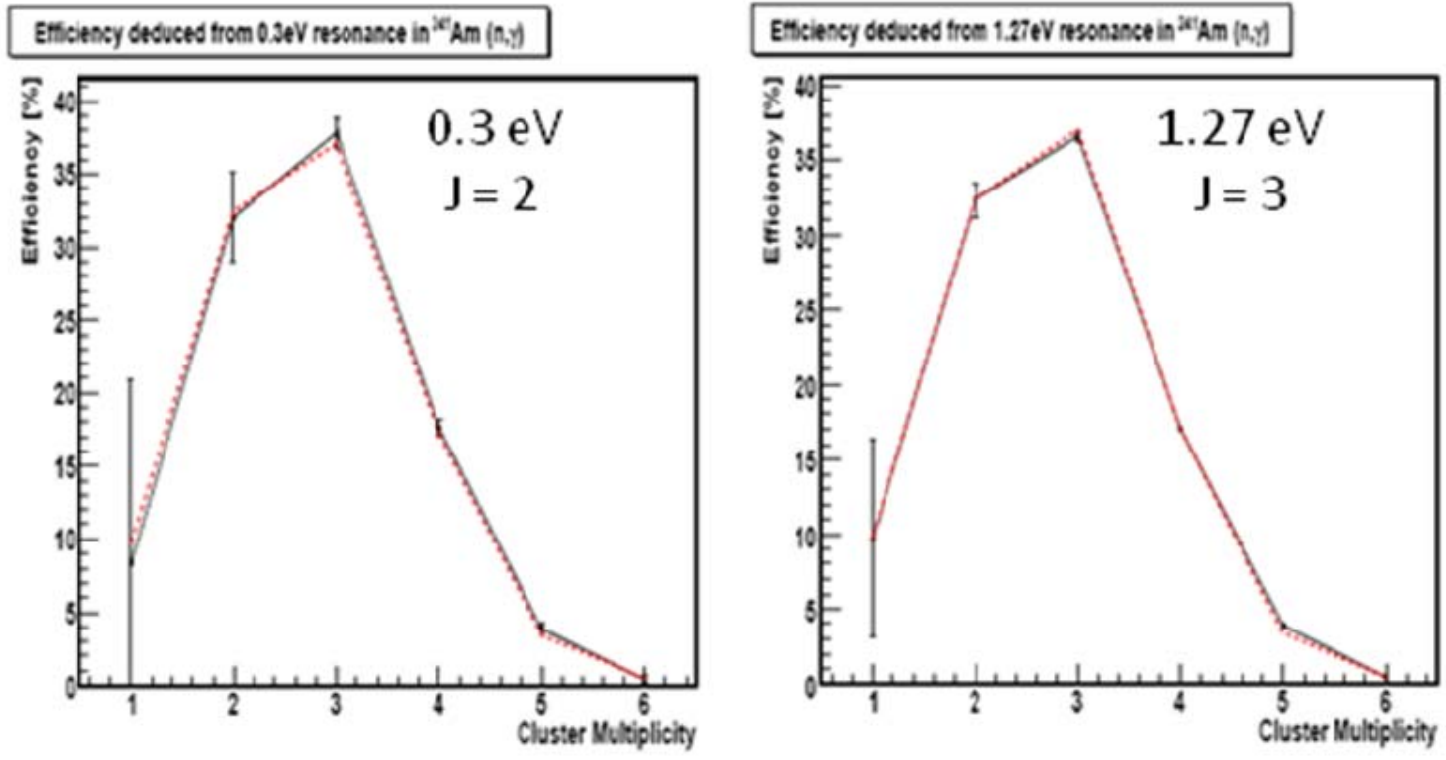

Figure 7. Monte-Carlo calculation of detection efficiency versus gamma multiplicity, compared to measured value, for 2 low-lying resonances in ${ }^{241} \mathrm{Am}(n, Y)$. 


\section{Conclusions}

Experiments with DANCE at the Lujan Center have shown that capture cross section data can be obtained on less than a milligram sample, although thicker targets are advantageous for cross sections above $1 \mathrm{keV}$. DANCE can provide good information on resolved resonances, and the "standard" samples studied are in good agreement with evaluations. In certain cases, the spin of the resonance can be determined by the gamma-ray multiplicity.

Measurements of capture cross sections to date have been normalized to thermal data or well-known resonances, although much effort is being directed to obtaining absolute cross sections. Measurements of capture in the continuum region on ${ }^{237} \mathrm{~Np}$ and ${ }^{236} \mathrm{U}$ are in substantial agreement with evaluations, but the ${ }^{234} U$ capture cross section was roughly $50 \%$ of the value recommended in ENDF/B-VI. Measure of the capture-to-fission ratios in actinides is under way using PPAC fission-tagging detectors as the target. In addition, work on gamma-ray strength functions and gamma spectra is beginning. Several papers are in press or nearing publication, and much more data is being analyzed.

\section{Acknowledgements}

The work was supported at Los Alamos and Livermore by Laboratory-directed research funds (LDRD) and programs in stockpile stewardship and advanced reactors. Efforts at North Carolina State University were supported by the Stockpile Stewardship Academic Alliance. This work made use of the moderated neutron beams at the Manuel J. Lujan Neutron Scattering center at the Los Alamos Neutron Science Center. Los Alamos National Laboratory is operated by Los Alamos National Security, LLC, for the National Nuclear Security Administration of the U.S. Department of Energy under contract DE-AC52-06NA25396.

\section{References}

[1] M. Heil, et al., A $4 \pi B a F_{2}$ detector for (n,Y) cross-section measurements at a spallation neutron source, Nucl. Instr. Meth A 459, 229-446 (2001).

[2] R. Reifarth, et al., Background identification and suppression for the measurement of (n,V) reactions with the DANCE array at LANSCE. Nucl. Instr. Meth. A 531, 530-543 (2004).

[3] G. Muhrer, private communication.

[4] Agilent Corporation, Acqiris Operation, Geneva Switzerland.

[5] J. Wouters, et al., Acquisition-Analysis System for the DANCE (Detector for Advanced Neutron Capture Experiments) $\mathrm{BaF}_{2}$ Gamma Ray Calorimeter. IEEE Transactions on Nuclear Science, 53, 880 (2006). 
[6] M.B. Chadwick, et al., ENDF/B-VII.0: Next Generation Evaluated Nuclear Data Library for Nuclear Science Technology, Nuclear Data Sheets 107, 2931-3060 (2006).

[7] T.A. Bredeweg, et al., Recent actinide nuclear data efforts with the DANCE $4 \pi B_{2} F_{2}$ array. Int'l. Conf.on Nucl. Data for Sci. and Tech 2007, to be published.

[8] E.-I. Esch, et al., The ${ }^{237} \mathrm{~Np}(\mathrm{n}, \gamma)$ cross section between $20 \mathrm{meV}$ and $500 \mathrm{keV}$. Submitted for publication.

[9] C. Coceva, et al., A method of spin assignment of neutron resonances based on capture gamma-ray detection. Nucl. Phys. A 117, 596-614 (1968). G. Georgiev et al., Determination of ${ }^{147} \mathrm{Sm}$ and ${ }^{148} \mathrm{Sm}$ resonance parameters. Nucl. Phys. A 565, 643-656 (1993).

[10] Yu. M. Gledenov et al., ${ }^{147} \mathrm{Sm}(\mathrm{n}, \alpha)$ cross section measurements from $3 \mathrm{eV}$ to $500 \mathrm{keV}$ : Implications for explosive nucleosynthesis reaction rates. Phys. Rev. C 62, 042801 (2000).

[11] P.E. Koehler, et al., Spin Measurements for $147 \mathrm{Sm}+n$ resonances: Further evidence for nonstatistical effects. Phys. Rev C 76, 025804 (2007).

[12] M. Jandel, et. al., GEANT4 simulations of the DANCE array. Nucl. Instr. Meth. In press (2007).

[13] R. Reifarth et al., Results of GEANT simulations and comparison with first experiments at DANCE: On how a not-quite $4 \pi$ array works. Los Alamos Report LA-UR-03-5560 (2003).

[14] F. Becvar, Simulation of Y cascades in complex nuclei with emphasis on assessment of uncertainties of cascade-related quantities. Nucl. Instru. Meth. A 417, 434-449 (1998).

[15] S. Agostinelli, et al., GEANT4 - a simulation toolkit. Nucl. Instr. Meth. A 506 (250-303) 2003. 